$L / D$ ratio. It can again be seen that an $L / D$ ratio of about 20 is required to insure constancy of the die swell ratio. The similarity in behavior of both the die swell and the exit pressure is to be expected in light of the fact that both are manifestations of the same elastic nature. It should be noted that the value of 20 diam. for the entrance length agrees quite favorably with the value of 15 found by Bagley (3) for another polyethylene, but not with that found by Metzner et al. (1).

\section{CONCLUSION}

On the basis of both die swell and exit pressure measurements, one is led to the conclusion that the entrance length required for the fully developed flow of polymer melts is quite large. It is further evident that the measurement of the pressure gradient (with currently available melt pressure transducers) is not sufficiently sensitive to respond to changes in the flow field that occur at distances from the entrance which are greater than that equivalent to about 1 capillary diam. Measurement of elastic properties such as exit pressure and die swell are apparently much better indicators of the development of the flow pattern. Of these, the die swell appears to be more sensitive and so should be used in the determination of the entrance length.

\section{NOTATION}

$c=$ constant in Equation (1)

$D$ = capillary diameter

$L_{*}=$ entrance length

$N_{R e}=$ Reynolds number

$P_{R, L}=$ exit pressure

$S_{r r}(R, L)=$ the total normal stress in the radial direction at the exit

\section{LITERATURE CITED}

1. Metzner, A. B., E. L. Carley, and I. K. Park, Modern Plastics, 37, No. 11, 133 (1960).

2. Bagley, E. B., S. H. Storey, and D. C. West, J. Appl. Polymer Sci., 7, 1661 (1963).

3. Kowalski, R. C., Ph.D. dissertation, Polytechnic Inst., Brooklyn, New York (1963).

4. Sakiadis, B. C., AIChE J., 8, 317 (1962).

5. Arai, Teikichi, paper presented at the Fifth International Congress of Rheology, Kyoto, Japan (Oct., 1968).

6. Mori, Yoshiro, and Kazumori Funatsu, Chemistry of High Polymers (Japan), 25, 391 (1968).

7. Han, C. D., Marvin Charles, and Wladimir Philippoff, Trans. Soc. Rheol., to be published.

8. Lipson, J. M., and A. S. Lodge, Rheologica Acta, 7, 364 (1968).

\title{
In Defense of the Crank-Nicolson Method
}

\section{J. O. WILKES}

\section{University of Michigan, Ann Arbor, Michigan}

A new finite-difference technique for solving the heat conduction equation has recently been published by Liu (1). To demonstrate the effectiveness of his method, Liu applied it to the problem $\partial u / \partial t=\partial^{2} u / \partial x^{2}$ subject to the initial condition $u(x, 0)=0,0 \leq x \leq 1$ and the boundary conditions $\partial u(0, t) / \partial x=0, u(1, t)=1, t>0$. For $\Delta x=0.05$ and $\Delta t=0.002$, corresponding to $\lambda=\Delta t /(\Delta x)^{2}$ $=0.8$, the results computed for $u$ were compared with the known analytical solutions and also with those obtained by the Crank-Nicolson (C-N) method, identified by Liu as "the classical implicit method." By taking the case that is the most favorable to Liu, the error involved at $t=0.5$ by using his "average" method is less by two or three orders of magnitude than the error involved using the C-N method, according to Liu (see lines 1 and 2 of Table 1).

This communication emphasizes that Liu's paper has, in any event, portrayed an unduly pessimistic picture of the accuracy of the $\mathrm{C}-\mathrm{N}$ method and, furthermore, that an almost trivial modification of the $\mathrm{C}-\mathrm{N}$ method (scheme $B$ below) yields results just as accurate as those generated by Liu's method.

TABLE $1.10^{5} \times$ ERror AT $t=0.5$, with $\Delta x=0.05$

\begin{tabular}{llrrrr} 
& \multicolumn{1}{c}{ Method } & $\lambda$ & $x=0.2$ & $x=0.6$ & $x=0.8$ \\
1 & C-N (Liu) & 0.8 & $1,767.0$ & 844.6 & 414.0 \\
2 & Liu (average) & 0.8 & 6.9 & 3.3 & 0.1 \\
3 & C-N (scheme A) & 0.8 & -91.5 & -56.6 & -29.8 \\
4 & C-N (scheme B) & 0.8 & -4.2 & -2.7 & -1.4 \\
5 & C-N (scheme B) & 5.0 & -3.0 & -2.1 & -0.6
\end{tabular}

I have reprogrammed the $\mathrm{C}-\mathrm{N}$ method for the same problem, using standard techniques [see (2), for example] with single-precision arithmetic on an IBM-360/67 computer. The question that arises, almost invariably, is what value of $u$ should be used in the approximation at $x=1$ and $t=0$. Strictly speaking (scheme A), according to the above mathematical statement of the initial and boundary conditions, the value $u=0$ should be taken (as was the case in Liu's paper), followed thereafter by $u=1$ at $x=1$ for $t>0$. Alternatively, as a numerical experiment (scheme B), one can also follow what is perhaps the more standard practice and try $u=1$ at $x=1$ for $t \geqslant 0$. There are other possibilities, of course, since we are dealing in terms of finite-difference approximations.

The results of my computations are summarized in lines 3,4 , and 5 of Table 1 . The error is defined here as the numerical approximation minus the exact value, which may differ in sign from Liu's definition as "the difference between the exact solution and the numerical solution."

My conclusions are evident: that the C-N errors according to Liu's programming are inordinately large, even compared with the results of Scheme $A$, and that with the slight modification according to scheme $\mathrm{B}$, the $\mathrm{C}-\mathrm{N}$ method gives results at least as good as those obtained by Liu's method, even (at this particular value of time) with a highly inflated time step.

\section{LITERATURE CITED}

1. Liu, S.-L., AIChE J., 15, 334-338 (1969).

2. Carnahan, B., H. A. Luther, and J. O. Wilkes, "Applied Numerical Methods," Wiley, New York (1969). 